\title{
UMA ANÁLISE DA VOLATILIDADE CONDICIONAL DA INFLAÇÃO MINEIRA NO PERÍODO PÓS-PLANO REAL ${ }^{1}$
}

\author{
Sidney Martins Caetano ${ }^{2}$ \\ Wilson Luiz Rotatori Correa
}

\begin{abstract}
Resumo - Este trabalho procurou investigar o comportamento da inflação na região metropolitana de Belo Horizonte via modelos ARCH, dada a sua importância relativa na composição do IPCA nacional. Os resultados, além de indicarem que a dinâmica da inflação mineira é determinada por um comportamento inercial, revelaram indícios de que os choques internos e externos sobre a economia brasileira levam certo tempo para afetar a dinâmica da volatilidade condicional da inflação mineira.
\end{abstract}

Palavras Chave: Inflação mineira, volatilidade, modelos ARCH.

\section{Introdução}

A inflação é definida por um processo de alta generalizada e contínua do índice geral de preços, que se traduz na redução gradativa do poder de compra interna da moeda ${ }^{4}$. Já a taxa de inflação é uma média da elevação dos preços em dado período. Como as oscilações nos preços não caminham na mesma direção e muito menos são de magnitudes idênticas, as alterações dos preços relativos são freqüentes em processos inflacionários, independente de sua magnitude.

Recebido em: 12/03/2008; Aceito em: 10/06/2008.

Doutor em Economia pela UFRGS, professor adjunto I da Universidade Federal de São João del Rei. E-mail: sidneymc@ufsj.edu.br

3 Doutor em Economia pela University of Southampton. Professor Adjunto I da Universidade Federal de São João del Rei. E-mail: wlrc@ufsj.edu.br

4 Quando, ao contrário, ocorre baixa generalizada e contínua do índice geral de preços, têm-se o conceito e o efeito inverso ao de inflação: deflação e aumento do poder de compra. 
O Brasil, nas décadas de 80 e 90, enfrentou problemas bastante graves ligados à inflação, e foi apenas a partir de meados da década de noventa que o país conseguiu "controlar" o processo de aceleração inflacionária com a introdução do plano real.

Se for considerada a definição clássica de Cagan (1956), o Brasil enfrentou apenas um período muito curto de hiperinflação, entre dezembro de 1989 e março de 1990. No entanto, se for utilizada a definição de inflação alta de Fisher et al. (2002), qual seja, aqueles períodos em que a inflação anual esteve acima de $100 \%$, terminando apenas quando essa taxa permaneceu abaixo de $100 \%$ por mais de um ano, então a economia brasileira esteve em regime de inflação alta por 15 anos e 2 meses, entre abril de 1980 e maio de 1995.

Após uma série de tentativas sem sucesso na Nova República, o Plano Real conseguiu baixar a inflação, que claramente foi mantida sob controle quando se refere especificamente à definição de inflação alta apresentada acima. No entanto, a área econômica dos governos não conseguiu impedir que, no decorrer desse período, alguns repiques inflacionários ocorressem e influenciassem, negativamente, a economia brasileira, como a desvalorização cambial (1999), a crise de confiança (2002/2003) e, em menor escala, em 2004, sem contabilizar os efeitos negativos gerados por crises externas, tais como a do México (1995); da Ásia (1997); a moratória na Rússia (1998); a queda da bolsa Nasdaq (2000); a crise na Argentina (2001), a crise política no Brasil (2005), entre outras.

Em um ambiente de aceleração inflacionária (elevação da inflação), principalmente em economias emergentes como a brasileira, alguns agentes econômicos ganham, enquanto muitos perdem. $\mathrm{O}$ repique inflacionário do segundo semestre de 2002 e primeiro de 2003, por exemplo, provocou significativa queda do poder de compra dos salários $(12,2 \%)$. Segundo Meirelles (2006), desde 1995, o único ano em que a parcela da renda dos $50 \%$ mais pobres não subiu foi justamente 2002 , que foi marcado por forte aceleração inflacionária. 
Portanto, um controle inflacionário também é objetivo indispensável para alcançar uma política social bem sucedida, uma vez que é a parte pobre da população a que mais sofre os efeitos perversos da inflação.

Podem-se citar outras conseqüências advindas de taxas de inflação elevada, como distorções na alocação de recursos da economia; instabilidade no balanço de pagamento, ao alterar o valor da moeda nacional e da taxa de câmbio real; incertezas a respeito dos investimentos privados, etc.

Conforme Romer (2005), a inflação pode gerar não apenas custos como também benefícios. Em termos de custos facilmente identificáveis, podese citar o fato de a inflação aumentar o custo de oportunidade de deter moeda, levando os agentes econômicos a querer deter menores quantidades desta, o que os obriga a efetuar trocas mais frequientes de outros ativos por moeda e implica os custos de "sola de sapato" "shoeleather costs"). Além disso os preços e os salários nominais têm de ser alterados com maior frequiência, ou a indexação é adotada. A inflação também distorce o sistema fiscal, tendendo, por exemplo, a aumentar a taxa efetiva de imposto aos rendimentos de capital.

Outros custos de uma inflação estável devem-se ao ajustamento defasado dos preços, o que leva a variações nos preços relativos. Isto dificulta a criação de relações de longo prazo entre empresas e clientes e tende a reduzir a eficiência nos mercados. Indivíduos e empresas podem ter dificuldade em contabilizar os efeitos da inflação, o que pode gerar decisões de investimento, de poupança ou de contração de empréstimos para compra de habitação ineficientes. A inflação também pode implicar custos de bem-estar, pelo simples fato de as pessoas não gostarem dela.

Em relação a custos de uma inflação variável, pode-se destacar o caso de muitos ativos estarem denominados em termos nominais, em que variações não antecipadas da inflação levam à redistribuição de riqueza. Como a inflação tende a ser mais variável quando é alta, maior inflação implica menor bem-estar. Engle (1982) procurou analisar a variância da 
inflação na Inglaterra por meio de um modelo alternativo aos usuais, em séries temporais.

Com dívidas denominadas em termos nominais, empresas e indivíduos tornam-se mais relutantes em empreender projetos de investimento, especialmente no longo prazo. Inflação alta e variável também pode desencorajar o investimento no longo prazo, por ser vista como um sintoma de um governo que funciona mal e que pode recorrer a nacionalizações, confiscos ou outros atos que prejudiquem os detentores de capital.

Como a inflação não é totalmente ruim, podem-se citar alguns de seus benefícios potenciais. Se o salário nominal for rígido, os salários reais serão mais facilmente ajustados se a inflação for mais alta. $\mathrm{O}$ governo pode gerar uma política macroeconômica mais flexível, pela opção de taxas reais de juros negativas. Uma economia com taxa média de inflação mais alta tem mais oportunidades de usar a política monetária para combater uma recessão, por exemplo. Inflação abaixo de determinado valor pode ser prejudicial à economia, razão por que alguma inflação (baixa a moderada) poderá ser desejável. A inflação também é fonte potencial de rendimento para o governo (senhoriagem). Em certas circunstâncias, poderá ser uma decisão ótima usar essa fonte de rendimentos a par dos impostos convencionais.

Nesse sentido, a despeito de não se enfrentar no Brasil um problema de inflação alta crônica, mas diante dos possíveis custos associados a esse fenômeno, torna-se relevante analisar o comportamento dos preços e a volatilidade condicional da inflação mineira, não só pela sua importância para o estado de Minas Gerais, como também por sua contribuição na composição do índice nacional de preços (IPCA), utilizado pelo Banco Central do Brasil (BACEN) no acompanhamento dos objetivos estabelecidos no atual sistema de metas para inflação.

O trabalho está distribuído da seguinte forma: introdução; seção 2, que mostra o comportamento da inflação mineira no período em análise; seção 3, que apresenta a metodologia utilizada; seção 4, que analisa as 
estatísticas descritivas e identifica a volatilidade condicional da taxa de inflação mineira; e, por último, a seção 5, que finaliza com a conclusão.

\section{Inflação na região metropolitana de Belo Horizonte}

Normalmente, os aumentos dos preços não ocorrem de forma sincronizada, ou seja, não há aumento idêntico do preço de todos os bens e serviços; desse modo, há um problema para calcular o tamanho da inflação. A forma como isso é resolvido é fazer uma média ponderada da elevação dos preços, tal como é feito para o IPCA (Índice de Preços ao Consumidor Amplo), calculado pelo Instituto Brasileiro de Geografia e Estatística (IBGE), por exemplo.

O período de coleta do IPCA estende-se, em geral, do dia $1^{\circ}$ a 30 do mês de referência, e sua população-objetivo abrange as famílias com rendimentos mensais compreendidos entre 1 e 40 salários-mínimos, qualquer que seja a fonte de rendimentos, e residentes nas áreas urbanas das regiões. A abrangência geográfica são as regiões metropolitanas de Belém (PA), Fortaleza (CE), Recife (PE), Salvador (BA), Belo Horizonte (MG), Rio de Janeiro (RJ), São Paulo (SP), Curitiba (PR) e Porto Alegre (RS), Brasília (DF) e município de Goiânia (GO), que cobrem extensa parte do território brasileiro e representam bem sua diversidade geográfica e econômica.

Dentro da estrutura de ponderação regional, em percentual, a região metropolitana de Belo Horizonte (RMHB) possui 9,15\% em 100\%. Em termos de significância, fica atrás da região metropolitana de São Paulo (RMAP) (36,26\%), Rio de Janeiro (RMRJ) (13,40\%) e Porto Alegre (RMPoA) (9,19\%). Uma nova estrutura de ponderação regional começou a vigorar a partir de julho de 2006; nesta nova estrutura, a RMBH passou à frente da RMPoA ${ }^{5}$.

Para maiores detalhes sobre esta nova estrutura de ponderação regional, ver Metodologia - Estrutura de ponderação a partir da POF 2000-2003. Disponível em:

http://www.ibge.gov.br/home/estatistica/indicadores/precos/inpc_ipca/srmipca_pof.pdf. 
A série de inflação mineira, considerada neste trabalho, refere-se ao período de 10/1994 a 12/2005. A Tabela 2.1. mostra que, na maior parte do período analisado, a taxa de inflação anual da RMBH supera a nacional, em valor absoluto. A elevação mais acentuada dos preços em Belo Horizonte, em relação à média nacional, pode ser reflexo, em parte, do aquecimento da economia mineira. Além disso, aspectos particulares nos mercados locais, bem como a rigidez na movimentação de fatores, podem ocasionar comportamento de preços específicos para cada região, em determinados segmentos. Nota-se também, na Tabela 2.1., que os esforços dos governos não foram suficientes para manter o indicador oficial de inflação (IPCA) próximo da meta, principalmente dentro do limite de tolerância fixado para os anos de 2001, 2002 e 2003.

Tabela 2.1 - Variação anual (\%) acumulada - IPCA Geral e RMBH 1995 a 2005

\begin{tabular}{|c|c|c|c|c|c|c|c|c|c|c|c|}
\hline & 1995 & 1996 & 1997 & 1998 & 1999 & 2000 & 2001 & 2002 & $2003^{17}$ & $2004^{11}$ & 2005 \\
\hline $\begin{array}{l}\text { IPCA } \\
\text { Goral }\end{array}$ & 22,41 & 9,56 & 5,22 & 1,65 & $\$, 94$ & 5,97 & 7,67 & 12,53 & 9,30 & 7,60 & 5,69 \\
\hline R M BH & 23,07 & 10,51 & 5,42 & $1,9 \mathrm{~s}$ & $\$, 02$ & 6,24 & 6,49 & $11,5 \mathrm{~s}$ & 11,18 & $\$, 93$ & 6,24 \\
\hline $\begin{array}{l}\text { Metas do } \\
\text { In flaçipe }\end{array}$ & & & & & $\begin{array}{c}8 \\
+\mu 2\end{array}$ & $\stackrel{6}{+/ 2}$ & $\stackrel{+}{+}$ & $\begin{array}{c}3,5 \\
+, 22\end{array}$ & $\begin{array}{c}3,25 \\
+f 2 \\
(4+f 2,5)\end{array}$ & $\begin{array}{c}3,75 \\
+/-2,5 \\
(5,5+-2,5)\end{array}$ & $\begin{array}{r}4,5 \\
+\quad 2,5\end{array}$ \\
\hline
\end{tabular}

Fonte: IBGE e BACEN. Elaboração dos autores. " A Carta Aberta, de 21/01/2003, estabeleceu metas ajustadas de 8,5\%, para 2003, e de 5,5\%, para 2004.

Devido aos choques em junho de 2002, a meta de inflação para 2003 foi alterada de 3,25\% para $4 \%$ a.a., bem como o intervalo de tolerância, ambos fixados em 2001. Em seguida, ocorreu ajustamento da meta de inflação para 8,5\%. Em junho de 2003, o Conselho Monetário Nacional $(\mathrm{CMN})$ adotou, para 2004, a meta ajustada pelo BACEN (5,5\%), com intervalo de tolerância de 2,5 p.p., e também fixou a meta de 2005 para $4,5 \%$ a.a. Esta última não foi alterada, mas o BACEN deixou claro, na condução da política monetária, que buscava atingir uma meta de 5,1\% ao ano. 
As atitudes tomadas para conter as altas de preços (por exemplo, aumento na taxa básica de juros - SELIC), influenciadas pelos choques internos e externos, acabaram por conter o crescimento econômico nacional.

Em 2005, por exemplo, o Produto Interno Bruto (PIB) de Minas Gerais acumulou crescimento de $4,7 \%$ no ano, em relação a 2004 , enquanto a economia brasileira teve um resultado anual de $2,3 \%$, bem inferior ao desempenho estadual. Segundo a Fundação João Pinheiro (2005),

dentre os principais fatores que mantiveram em alta $o$ nível de atividades da economia mineira em 2005, encontram-se o maior volume de recursos liberados para obras, em particular, as viárias e de infraestrutura, o modesto desempenho do mercado interno e do crédito com os empréstimos em consignação. Alia-se a estes o dinamismo do setor exportador, conseguindo saltos vantajosos, não obstante o câmbio apreciado e a normalidade da economia internacional, com seus mercados sempre receptivos aos produtos mineiros.

No caso da economia nacional, em 2005 esta foi "puxada" por componentes relacionados com comércio exterior, enquanto o principal componente, o investimento, que define a dinâmica efetiva da economia, cresceu cerca de apenas $1,7 \%$.

A Figura 2.1 apresenta a evolução mensal da taxa de inflação brasileira e da região metropolitana de Belo Horizonte, para o período de 10/1994 a 12/2005, o que representa parcialmente os períodos pré e pós-regime de metas para inflação.

Visualmente, nota-se nas séries de inflação uma tendência de queda semelhante após a implantação do Plano Real até o final de 1996. A partir de 1997, percebe-se certa estabilidade das taxas, já que a média 
da série estabilizou-se entre 0 e $1 \%$, mas com alguns repiques inflacionários, sendo o mais pronunciado destes entre meados de 2002 a julho de 2003. Não obstante, a despeito dessa maior estabilidade na média da taxa, observa-se certa volatilidade principalmente como efeito dos repiques observados.

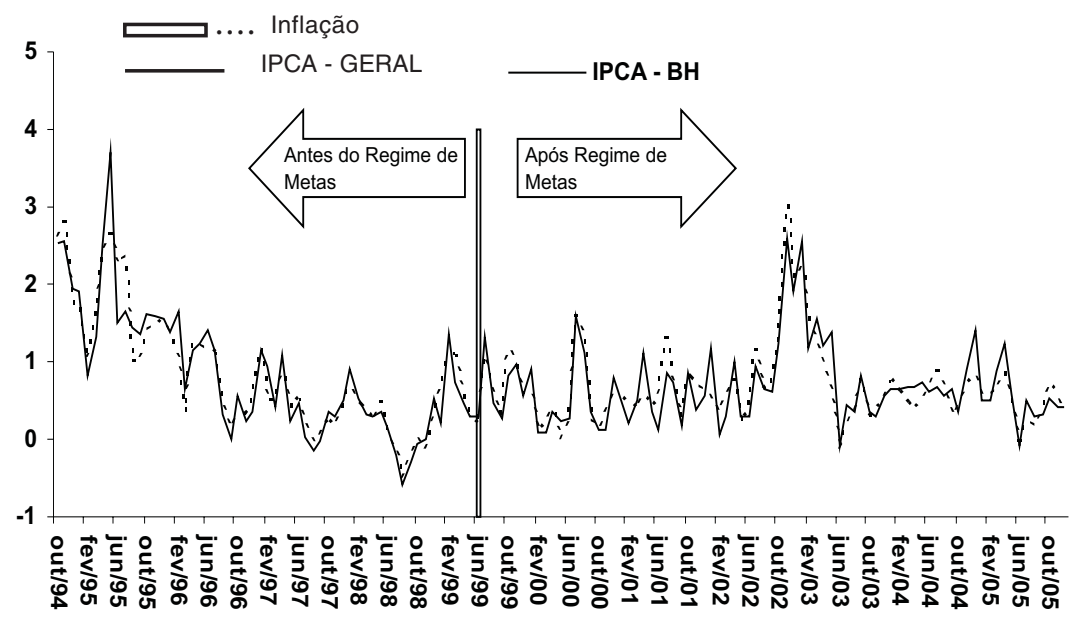

Figura 2.1 -Evolução mensal da taxa de inflação (IPCA \%) nacional e $\mathrm{RMBH}$

Os períodos de alta volatilidade costumam coincidir com épocas nas quais ocorrem crises tanto internas quanto externas. Como em ambos os períodos pré e pós-regimes de metas para inflação houve a ocorrência de crises, o primeiro mais que o segundo, então é interessante tentar modelar a volatilidade condicional da inflação da RMBH e observar seu comportamento nesses momentos. No entanto, antes de modelar a volatilidade condicional, elabora-se uma análise mais simples, ao comparar e avaliar a volatilidade não-condicional antes e depois do regime de metas para inflação. 
A Tabela 2.2. mostra que, após a introdução do regime de metas para inflação, a volatilidade não-condicional da taxa de inflação nacional reduziuse de 0,91 para 0,68 , considerando o mesmo intervalo de tempo para ambos os períodos. Ao levar em conta o período pós-regime de metas como um todo, nota-se uma queda de 0,64 na volatilidade.

No caso da taxa de inflação da RMBH, pode-se observar que sua volatilidade registrou queda de 0,91 para 0,63 . Se for considerado o período pós-regime, a queda foi ainda maior, 0.57. A média mensal da inflação segue o mesmo comportamento da volatilidade não-condicional para ambas as séries.

Tabela 2.2. Comparação antes e depois do regime de metas para inflação

\begin{tabular}{|c|c|c|c|}
\hline & $\begin{array}{c}\text { Antes (1994.4 - } \\
1999.2)\end{array}$ & $\begin{array}{c}\text { Depois (1999:3 - } \\
\text { 2004:1) }\end{array}$ & $\begin{array}{c}\text { Depois (1999:3 - } \\
2005: 4)\end{array}$ \\
\hline \multicolumn{4}{|c|}{ Volatilidades - Coeficiente de Variação(des vio-padrãómédia, dados trimestrais) } \\
\hline Indice Geral(IFCA) & 0,91 & 0,68 & 0,64 \\
\hline RM. Belo Horizonte & 0,91 & 0,63 & 0,57 \\
\hline \multicolumn{4}{|c|}{ Média Mersal da Irflaçăo (\%) } \\
\hline Índice Geral(IFCA) & 0,83 & 0,71 & 0,66 \\
\hline RM. Belo Horizonte & 0,85 & 0,71 & 0,68 \\
\hline
\end{tabular}

Fonte: IBGE. Elaboração dos autores.

A Tabela 2.3. mostra que mais da metade da população ocupada mineira possui rendimento até dois salários mínimos. Num ambiente de volatilidade do nível de preços, torna-se difícil manter determinado nível de aquisição de bens e serviços necessários e ainda conseguir poupar e,ou, protegerse dos aumentos constantes de preços, sem contabilizar as incertezas e os efeitos destas sobre a economia, como destacado anteriormente.

Portanto, a estabilidade de preços mostra-se relevante no caso de Minas Gerais e é uma condição necessária, mas não suficiente, para que este Estado consiga taxas de crescimento maiores, de forma a aumentar o bem-estar da sociedade. 
Tabela 2.3 -População ocupada (1), segundo classes de rendimentos (2) $-\mathrm{MG}-2001$ a 2003

\begin{tabular}{|c|c|c|c|c|c|c|}
\hline Especificaçăo & 2001 & (\%) & 2002 & $(\%)$ & 2003 & $\%)$ \\
\hline Minas Gerais & 8.335 .272 & $100,00 \%$ & 8.737068 & $100,00 \%$ & 8.835 .291 & $100,00 \%$ \\
\hline Áté I SM & 2.377 .007 & $28,53 \%$ & 2.739840 & $31,36 \%$ & 2.899 .733 & $32,82 \%$ \\
\hline De la $2 \mathrm{SM}$ & 2.290 .168 & $27,48 \%$ & 2.400034 & $27,47 \%$ & 2.393 .945 & $27,10 \%$ \\
\hline De 2 a 5 SM & 1.700 .150 & $20,40 \%$ & 1.614 .739 & $18,48 \%$ & 1.696 .752 & $19,20 \%$ \\
\hline De 5 a $10 \mathrm{SM}$ & 561.306 & $6,73 \%$ & 508.375 & $5,82 \%$ & 421.494 & $4,77 \%$ \\
\hline De 10 a $20 \mathrm{SM}$ & 213.447 & $2,56 \%$ & 198.286 & $227 \%$ & 185.317 & $2,10 \%$ \\
\hline Mais de $20 \mathrm{SM}$ & 78.100 & $0,94 \%$ & 80.522 & $0,92 \%$ & 69.776 & $0,79 \%$ \\
\hline $\begin{array}{l}\text { Sem rendimentos e } \\
\text { sem declaraçäo (3) }\end{array}$ & 1.114 .285 & $13,37 \%$ & 1.195272 & $13,68 \%$ & 1.168 .274 & $13,22 \%$ \\
\hline
\end{tabular}

Fonte: FJP (2005); Dados Brutos da PNAD; (1) pessoas de 10 anos e mais de idade; (2) SM - Rendimentos em Salário Mínimo; (3) Inclusive pessoas que receberam somente em benefícios.

Em ambiente de relativa incerteza, o uso de técnicas estatísticas e econométricas que permitam a determinação de padrões de volatilidade das taxas de inflação no estado torna-se, portanto, um instrumento indispensável à categorização do desenvolvimento regional e à determinação de políticas públicas que visem à diminuição de desigualdades regionais.

\section{Metodologia: modelos ARCH}

Neste estudo, o objetivo principal é modelar o que se chama de volatilidade, que é variância condicional de uma variável; no caso, a taxa de inflação mineira medida pela variação do IPCA da região metropolitana de Belo Horizonte. Para isso, utilizam-se modelos que apresentam a variância condicional que evolui no tempo.

Os modelos ARCH (AutoRegressive Conditionally Heteroscedastic Model), ou modelos auto-regressivos com heterocedasticidade condicional, foram introduzidos por Engle (1982), com o objetivo de estimar a variância da inflação na Inglaterra por meio de um modelo alternativo 
aos usuais em séries temporais. A idéia básica é que a taxa de inflação seja não-correlacionada serialmente e que a volatilidade (variância condicional) seja uma função linear dos choques ao quadrado, ocorridos no passado da taxa.

Bollerslev (1986) ampliou o trabalho original de Engle, ao desenvolver uma técnica que permite a inclusão de valores defasados da variância condicional na equação da variância corrente. $\mathrm{O}$ modelo $\operatorname{ARCH}(p, q)$ generalizado, que viria a ser chamado $\operatorname{GARCH}(p, q)$, permite a presença de componentes auto-regressivos e média móvel na variância heterocedástica ${ }^{6}$. Esse processo GARCH pode representar um processo $\mathrm{ARCH}$ de mais alta ordem, de forma parcimoniosa.

Um modelo GARCH $(1,1)$ especifica que a variância depende dos valores passados da variável dependente e do quadrado dos erros passados da regressão original, conforme a equação (3.1), a seguir:

$$
\sigma_{t}^{2}=\beta_{0}+\beta_{1} \varepsilon_{t-1}^{2}+\beta_{2} \sigma_{t-1}^{2} \text {. }
$$

O modelo GARCH captura, com sucesso, os fatos de a série ter caudas espessas e de um aumento de volatilidade persistir por determinado tempo, mas peca quando procura captar os efeitos de alavancagem, ou seja, os modelos GARCH são incapazes de captar assimetrias nas taxas ou retornos, como ocorre na área financeira. Além disso, os parâmetros do modelo têm que ser positivos, para que a estacionariedade das séries seja mantida.

$\mathrm{Na}$ tentativa de resolver essas dificuldades, Nelson (1991) propôs o modelo de heterocedasticidade condicional auto-regressivo generalizado exponencial (EGARCH), que permite a absorção dos impactos assimétricos e a possibilidade irrestrita de que alguns coeficientes sejam negativos.

\footnotetext{
6 Um resumo da literatura sobre modelos GARCH pode ser obtido em Bueno (2002). Ver, também, Morettin e Toloi (2004).
} 
Alguns autores, como Nelson (1991), argumentaram que, para algumas séries financeiras, a volatilidade subseqüente a um choque negativo seria maior que a gerada por um choque positivo. Esse fenômeno ficou conhecido, na literatura, como leverage effect (efeito de alavancagem). No modelo EGARCH, a especificação para a volatilidade condicional torna-se:

$\log \sigma_{t}^{2}=w+\beta \log \sigma_{t-1}^{2}+\alpha\left|\frac{\varepsilon_{t-1}}{\sigma_{t-1}}\right|+\gamma \frac{\varepsilon_{t-1}}{\sigma_{t-1}}$.

A especificação logarítmica não permite que a variância seja negativa, o que evita a imposição de restrições aos sinais dos parâmetros. A presença do leverage effect pode ser testada pela hipótese de que $\gamma>0$. O impacto será assimétrico se $\gamma \neq 0^{7}$. Um parâmetro $\gamma$ negativo implica que a volatilidade das taxas de inflação aumentará quando os choques forem negativos.

Outro modelo que visa captar essa assimetria dos choques na volatilidade é o TARCH ou Threshold ARCH, introduzido por Zakoian (1994) e Glosten, Jaganathan e Runkle (1993). A especificação para a variância condicional é dada por:

$\sigma_{t}^{2}=w+\alpha \varepsilon_{t-1}^{2}+\gamma \varepsilon_{t-1}^{2} d_{t-1}+\beta \sigma_{t-1}^{2}$

em que $d_{t}=1$ se $\varepsilon_{t}<0$, e 0 , caso contrário.

Neste modelo, choques positivos $\left(\varepsilon_{t}>0\right)$ e negativos $\left(\varepsilon_{t}<0\right)$ possuem diferentes efeitos na volatilidade condicional - choques positivos tem um

\footnotetext{
Nelson (1991) propõe a função de distribuição Erro Generalizado (GED) para estimar o EGARCH, enquanto o EViews admite que os erros tenham distribuição normal. Detalhes sobre as diferenças entre a especificação do EViews para o modelo EGARCH e a do modelo original de Nelson, ver EViews 4 User's Guide (2002, pág. 393-394).
} 
impacto em , enquanto o choque negativo impacta em $(\alpha+\gamma)$. Se $\gamma>0$, então o efeito alavancagem existirá; se $\gamma \neq 0$, o impacto será assimétrico. Uma especificação de ordem maior, para o modelo TARCH, pode ser apresentada por

$$
\sigma_{t}^{2}=w+\sum_{i=1}^{q} \alpha_{i} \varepsilon_{t-i}^{2}+\gamma \varepsilon_{t-1}^{2} d_{t-1}+\sum_{j=1}^{p} \beta_{j} \sigma_{t-j}^{2} .
$$

Após esses breves comentários teóricos, a próxima seção visa identificar a dinâmica da volatilidade condicional da taxa de inflação da região metropolitana de Belo Horizonte, entendida, aqui, como a inflação mineira.

\section{Resultados empíricos}

Inicia-se esta seção com o estudo do comportamento estatístico da série de inflação mineira, referente ao período 10/94 até o final de 2005.

Uma das suposições de parte dos modelos econométricos é a de que as séries modeladas sejam estacionárias. Para verificar essa hipótese na inflação mineira, implementou-se o teste ADF (Aumented Dickey-Fuller). Ao aplicar, de forma complementar, o teste de raiz unitária com presença da tendência, os resultados mostraram, como era de esperar, que em nenhuma das equações de teste a tendência foi significativa ${ }^{8}$. A Tabela 4.1 mostra, por meio dos valores calculados, a rejeição da hipótese nula de presença de raiz unitária.

\footnotetext{
8 As defasagens, definidas automaticamente, são introduzidas para controlar problemas de autocorrelação e, por sua vez, foram determinadas através dos critérios de seleção Schwartz (SIC).
} 
Tabela 4.1 - Teste de Raiz Unitária - Augmented Dickey-Fuller

\begin{tabular}{llcccc}
\hline Variável & Mode lo & Lags & t- estatístico & $1 \%{ }^{*}$ & $5 \%$ \\
& Trend e intercept & 0 & $-5.843465^{* * * *}$ & -4.027959 & -3.443704 \\
AIPCABH & Intercept & 0 & $-5.745191^{* * * *}$ & -3.479656 & -2.883073 \\
& None & 2 & $-2.700207^{* * * *}$ & -2.582599 & -1.943266 \\
\hline
\end{tabular}

Obs.: ***, $* *$ significativo a $1 \%$ e $5 \%$, respectivamente; a $\mathrm{P}$ Valores Críticos. Critério de seleção SIC, lag máximo $=12$.

As estatísticas descritivas da inflação mineira e nacional, Tabela 4.2., revelam que no período analisado, ou seja, em uma ambiente de certa estabilidade de preços (baixa inflação), as inflações médias, medianas e seus respectivos desvios-padrões tiveram comportamentos bastante similares.

Tabela 4.2 - Estatísticas descritivas

\begin{tabular}{lccccc}
\hline & Média & Mediana & Máximo & Mínimo & Desvio padrăo \\
\hline IPCA Geral & 0.73 & 0.57 & 3.02 & -0.51 & 0.6425 \\
IPCA RMIBH & 0.75 & 0.56 & 3.71 & -0.58 & 0.6706 \\
\hline & Simetria & Curbse & Jarque-Bera & P-value & \\
\hline IPCA Geral & 1.395 & 5.098 & 68.555 & 0.000 & \\
\hline IPCA RMIBH & 1.328 & 5.611 & 78.047 & 0.000 & \\
\hline
\end{tabular}

Obs.: Valores estimados pelos autores no Eviews 4.1. Número de observações = 135.

Os dados mineiros também revelaram que sua volatilidade não-condicional é pouco superior à nacional. Se a taxa de inflação apresentasse variância condicional e evoluísse no tempo, esta seria uma característica de que os modelos não-lineares são mais adequados para descrever tal comportamento. Com maior interesse neste último ponto, a próxima seção procura modelar a dinâmica da volatilidade condicional da taxa de inflação mineira. 
O primeiro passo consiste em ajustar um modelo $\operatorname{ARMA}(p, q)$ à série de taxa de inflação da RMBH, para remover a correlação serial entre as observações. Após definir a equação da média, verifica-se presença de heterocedasticidade condicional, utilizando-se os testes de Box-PierceLjung ou teste de Multiplicadores de Lagrange (ML) .

A identificação das ordens, em geral, é feita pela função de autocorrelação (fac) e pela função de autocorrelação parcial (facp), pois a análise dessas funções indica a existência de dependência linear entre as observações e, portanto, fornece indícios de qual modelo seria o mais apropriado.

Uma análise da facp amostral indica um modelo auto-regressivo como o mais apropriado. O indício é de que seja um modelo AR(1). No entanto, a adequação deste modelo também é testada ao verificar outros critérios de ajustamento para modelos auto-regressivos. O critério de informação, de Schwartz (SIC), confirma o modelo AR(1), mas o critério de informação de Akaike (AIC) indica um modelo AR(3).

A equação (4.1.1) apresenta o ajustamento do modelo AR(3), pelo que se verifica a existência de coeficientes não-significativos, a um nível de $5 \%$, pela estatística $t$ entre parênteses.

$\Pi_{\mathrm{t}}=0.6746+0.4968 \Pi_{\mathrm{t}-1}+0.0435 \Pi_{\mathrm{t} \cdot 2}+0.1494 \Pi_{\mathrm{t} \cdot 3}+\mathrm{a}$
(4.8038)
(5.6872)
$(0.4483)$
(1.7708)

Ao eliminar esses coeficientes, obtém-se o modelo AR(1), antes selecionado pelo critério $\mathrm{SIC}^{10}$ :

\footnotetext{
9 Ver Morettin e Toloi (2004).

10 Também foi analisado um modelo ARMA(1,1). Apesar de apresentar coeficientes significativos, este modelo não demonstrou melhor adequação segundo os critérios AIC e SIC.
} 
$\Pi_{\mathrm{t}}=0.7144+0.6271 \Pi_{\mathrm{t}-1}+\mathbf{q}$

\section{$(6,1142) \quad(9.6632)$}

A análise de resíduos do modelo (4.1.2) fornece $\mathrm{Q}(20)=25,216$, com Pvalor igual a 0,154 , o que demonstra ter eliminado a correlação serial da série. O próximo passo é verificar se os resíduos desse modelo apresentam heterocedasticidade condicional. A fac indica a existência de heterocedasticidade, fato também confirmado pelo teste ML $(\mathrm{T}=13,4654$ com $\mathrm{P}=0,0092$, para $\mathrm{p}=4)$. Enquanto isto, a facp sugere um ARCH(1). Os resultados do ajustamento deste modelo mostram a significância dos parâmetros.

Portanto, o modelo final pode ser expresso da seguinte forma:

$$
\begin{aligned}
& \Pi_{\mathrm{t}}=0,6622+0,6158 \Pi_{\mathrm{t}-1}+\mathbf{q}, \\
& \sigma_{t}^{2}=0,1750+0,2918 \varepsilon_{t-1}^{2} .
\end{aligned}
$$

Este modelo ajustado define o comportamento da volatilidade condicional da taxa de inflação da RMBH como uma função linear dos choques ao quadrado, ocorridos no passado desta série, em apenas 1 (uma) defasagem.

As fac e facp dos resíduos padronizados juntamente com as estatísticas de Ljung-Box, com p-valores maiores ou iguais a 0,017 , não indicaram uma boa adequação do modelo para modelar a dependência linear entre taxas sucessivas.

Já as fac, facp e as estatísticas de Ljung-Box dos quadrados dos resíduos padronizados mostraram que não existe heterocedasticidade condicional nos resíduos do modelo. $\mathrm{O}$ teste $\mathrm{ML}(\mathrm{T}=3,6529 \operatorname{com} \mathrm{P}=0,4550$, para $\mathrm{p}=$ 4) confirma também esse resultado.

"A identificação da ordem de um modelo GARCH, a ser ajustado a uma série real, usualmente é difícil. Recomenda-se que se usem modelos de 
ordem baixa, como $(1,1),(1,2)$ ou $(2,1)$, e depois se escolha o modelo com base em vários critérios, como AIC ou BIC," (Morettin e Toloi, 2004). O uso desses modelos para tentar minimizar o problema da dependência linear acima não adiantou, pois os parâmetros dos modelos ajustados não foram significativos.

Uma alternativa foi estimar modelos EGARCH e TARCH, já que ARCH e GARCH tratam, simetricamente, as variáveis. A idéia aqui é reanalisar a taxa de inflação da RMBH pelo uso de modelos que permitem verificar se um efeito assimétrico deve ser incorporado aos modelos ajustados anteriormente.

A partir disto, foram selecionados e estimados os modelos EGARCH (1, 1) e TARCH $(1,1)$ com base nos critérios AIC e SIC. Os resultados são contraditórios, pois o modelo EGARCH $(1,1)$ mostrou que o efeito assimétrico não deve ser incorporado ao modelo, enquanto o TARCH $(1,1)$ indicou a existência de um impacto de informação assimétrica.

Com o objetivo de comparar os modelos ajustados às taxas de inflação da RMBH, calcularam-se alguns critérios de ajustamento (Tabela 4.1.1), bem como alguns testes de especificação para os resíduos.

Ao analisar os resultados da Tabela 4.1.1. pode-se dizer que o modelo AR (1)-TARCH $(1,1)$ ajustou-se melhor à série, pois apresentou os melhores valores para os critérios de seleção, como também os melhores testes de especificação, em conjunto, para os resíduos ao quadrado estimados. 
Tabela 4.1.1 - Valores de alguns critérios de ajustamento e testes de especificação

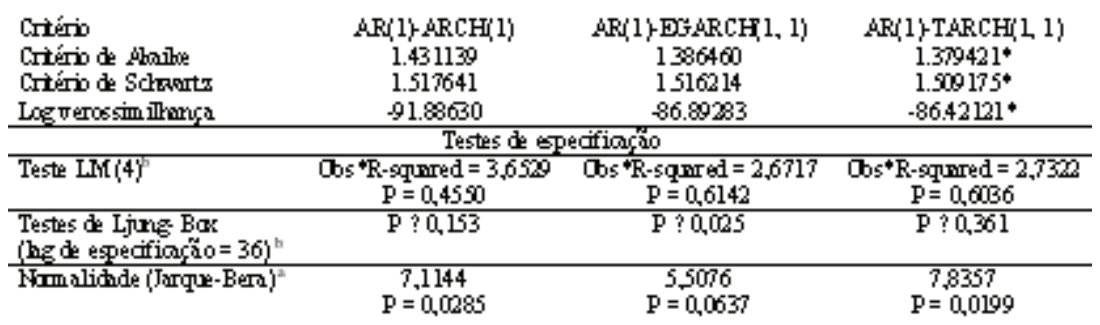

Obs.: (a) teste para os resíduos padronizados, (b) teste para o quadrado dos resíduos padronizados.

Assim, a Figura 4.1.1 apresenta a estimativa do desvio-padrão condicional para este último modelo.

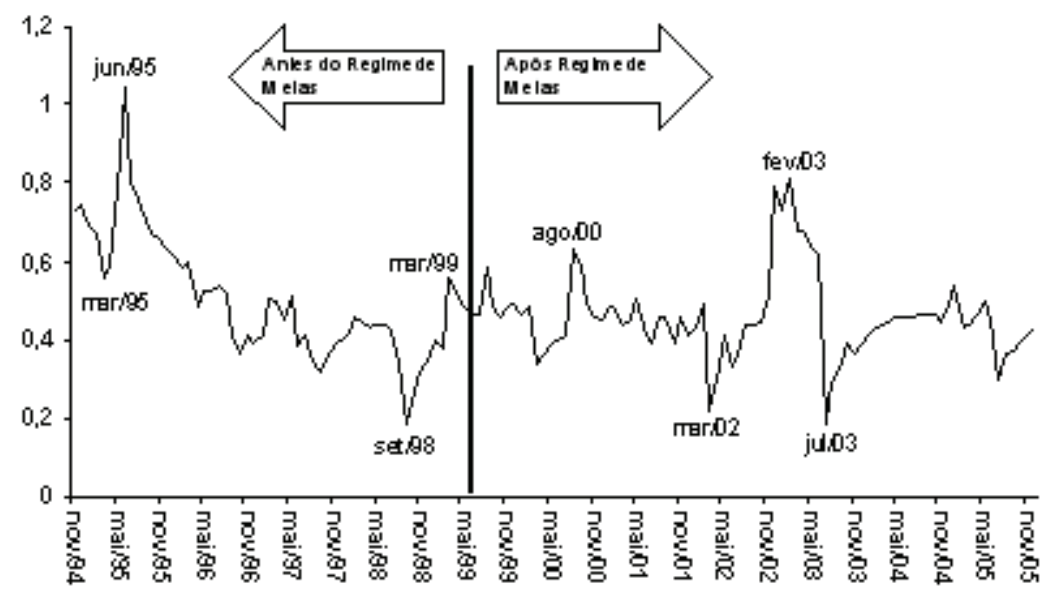

Figura 4.1.1 - Estimativa do desvio-padrão condicional da taxa de inflação da RMBH

Ao analisar a dinâmica da volatilidade da inflação mineira, observa-se que os maiores picos (em ordem cronológica) na volatilidade condicional 
estimada foram precedidos por crises internas e externas, como em junho de 1995, após a economia já sentir os efeitos negativos da crise mexicana; em outubro de 1997, quando houve certa alta depois do período final da crise da Ásia; em agosto de 1998, quando houve elevação da volatilidade pós-moratória na Rússia; e em janeiro de 1999, com a crise cambial brasileira, antes da implantação do regime de metas de inflação. Posteriormente, esse comportamento continuou o mesmo frente às novas crises, dada a queda da Bolsa Nasdaq, em abril de 2000, e dada a crise de confiança na economia brasileira, em 2002.

Assim, os períodos de alta volatilidade da taxa de inflação da RMBH parecem não coincidir com épocas nas quais ocorreram choques na economia, apresentando certo atraso no impacto desses choques, diferentemente do observado em algumas séries econômicas, principalmente financeiras, nas quais estes são coincidentes.

\section{Conclusão}

Este trabalho procurou analisar, via estatísticas descritivas e modelos ARCH, o comportamento da inflação na região metropolitana de Belo Horizonte/MG, dada a sua importância relativa na composição do índice nacional de preços (IPCA).

As estatísticas descritivas mostraram certa redução de volatilidade na inflação mineira e nacional a partir da implementação do sistema de metas para inflação. No entanto, cabe destacar que a introdução do regime de metas não pode ser considerada unicamente como responsável por esse comportamento decrescente. A redução observada na inflação não foi um fenômeno observado apenas no Brasil. Outros fatores mundiais favoráveis, como a onda deflacionista observada com as exportações de produtos manufaturados de países do sudeste asiático, principalmente a China, foram importantes para a redução da inflação em diferentes países. Particularmente no Brasil, a abertura econômica, associada à maior liquidez nos mercados financeiros internacionais e à liberalização do mercado de 
capitais, permitiu o financiamento de crescentes volumes de importação, e o país pôde aproveitar, em maior escala, a deflação observada em nível mundial.

Observou-se também que a taxa de inflação anual da RMBH superou a nacional, em valor absoluto. Apesar de essa elevação mais acentuada dos preços em Belo Horizonte ter sido especulada no decorrer das análises, tal fato deve ser analisado com maior rigor estatístico, pois testes estatísticos podem mostrar equivalência entre as séries, bem como comportamento homogêneo em relação às demais regiões.

Já a dinâmica da média e da volatilidade condicional, estimada para a inflação mineira, demonstraou ter comportamento inercial e assimétrico, respectivamente. Os resultados revelaram que os choques internos e externos na economia brasileira levam certo tempo para afetar a dinâmica da volatilidade condicional da inflação mineira, ou seja, o modelo AR(1)$\operatorname{TARCH}(1,1)$ selecionado revela que choques defasados um período e a volatilidade do período anterior afetam a volatilidade da inflação mineira corrente.

\section{Referências}

BUENO, R. L. S. Ainda os modelos GARCH. Revista Economia Aplicada, v. 6, n. 2. 2002.

BOLLERSLEV, T. Generalized autoregressive conditional heteroskedasticity. Journal of Econometrics, 31, 307-327, 1986.

CAGAN, P. The monetary dynamics of hyperinflation, in Friedman, M. (ed) Studies in the Quantity Theory of Money, Chicago: University of Chicago Press, 1956.

ENGLE, R. F. Autoregressive conditional heteroskedasticity with estimates of the variance of U. K. inflation. Econometrica, 50, 9871008, 1982. 
EVIEWS Versão 4.1. Quantitative Micro Software. 2002.

FISHER, S., et al. Modern hyper- and high inflations. Journal of Economic Literature, XL (3), 2002.

FUNDAÇÃO JOÃO PINHEIRO - FJP. Perfil de Minas Gerais 2005. Disponível em: http://www.fjp.gov.br/produtos/cei/ perfil2005portugues.pdf. Acesso em: 21/03/2006.

GLOSTEN, L.; JAGANNATHAN; RUNKLE, D. On the relation between expected value and the volatility of the nominal excess return on stocks. Journal of Finance, 45, 1779-1801, 1993.

HOLANDA, M. C. Distribuição regional de inflação e o regime de metas de inflação no Brasil. Revista Econômica do Nordeste, Fortaleza, v.32, n. especial, p. 458-466, 2001.

IBGE - Metodologia - Estrutura de ponderação a partir da POF 20002003. Disponível em: http://www.ibge.gov.br/home/estatistica/ indicadores/precos/inpc ipca/srmipca pof.pdf. Acesso em: 21/03/2006.

MEIRELLES, H. C. Implementação da Política Monetária no Brasil. Disponível em: http://www.bc.gov.br/?APRESPRONUNC. Acesso em: 25/03/2006.

MORETTIN, P. A.; TOLOI, C. M. C. Análise de series temporais. São Paulo: Edgard Blücher, 2004.

NELSON, D. B. Conditional heteroskedasticity in asset returns: A new approach. Econometrica, 59, 347-70, 1991.

ROMER, D. Advanced Macroeconomics. $3^{\mathrm{a}}$ edição, McGraw-Hill, New York. 2005.

ZAKOIAN, J. M. Threshold heteroskedasticity models. Journal of Economic Dynamics and Control, 18, 931-955, 1994. 
Abstract - In this paper we dedicate attention to the inflation dynamics in the metropolitan area of Belo Horizonte - Minas Gerais - through estimating an ARCH model considering its relative significance among those metropolitan areas that compose the national consumer price index (IPCA). Our results indicate that inflation in Minas Gerais presents an inertial component and that shocks hitting the Brazilian economy independent of arising from internal or from external sources only affect the conditional inflation volatility in Minas Gerais with a delay.

Keywords: Regional inflation, volatility, ARCH models. 